\title{
VIII JORNADA UNIVERSITARIA DE DESARROLLO CIENTÍFICO (JUDC) 2013 \\ Facultad de Humanidades y Ciencias Jurídicas
}

Dedicada a la juventud emprendedora

Gloria Argentina López Alvarado*

\section{INFORME DE LA VIII JORNADA FACULTATIVA DE DESARROLLO CIENTÍFICO (JUDC) DEDICADA A LA JUVENTUD EMPRENDEDORA}

E n el marco del XXXII Aniversario de la institucionalización de la Jornada Universitaria de Desarrollo Científico (JUDC) en la UNAN-Managua y la VIII Jornada de la Facultad de Humanidades y Ciencias Jurídicas dedicada a la JUVENTUD EMPRENDEDORA, se observo un buen ritmo de participación, planificación y entusiasmo en la comunidad estudiantil aceptando el reto de incursionar en este quehacer investigativo, para ir fortaleciendo su desempeño con creatividad, innovación y emprendedurismo.

En esta Jornada se involucraron los Departamentos adscritos a la Facultad en el siguiente orden: Antropología, Historia, Derecho, Geografía, Psicología, y Filosofía Además la Coordinación de las carreras de Filología y Comunicación, Trabajo Social; Diplomacia y Ciencias Políticas.

En cada una de estas instancias académicas, hubo planificación y seguimiento en el proceso orientador y de acompañamiento a los estudiantes proyectistas, para la presentación de trabajos investigativos,

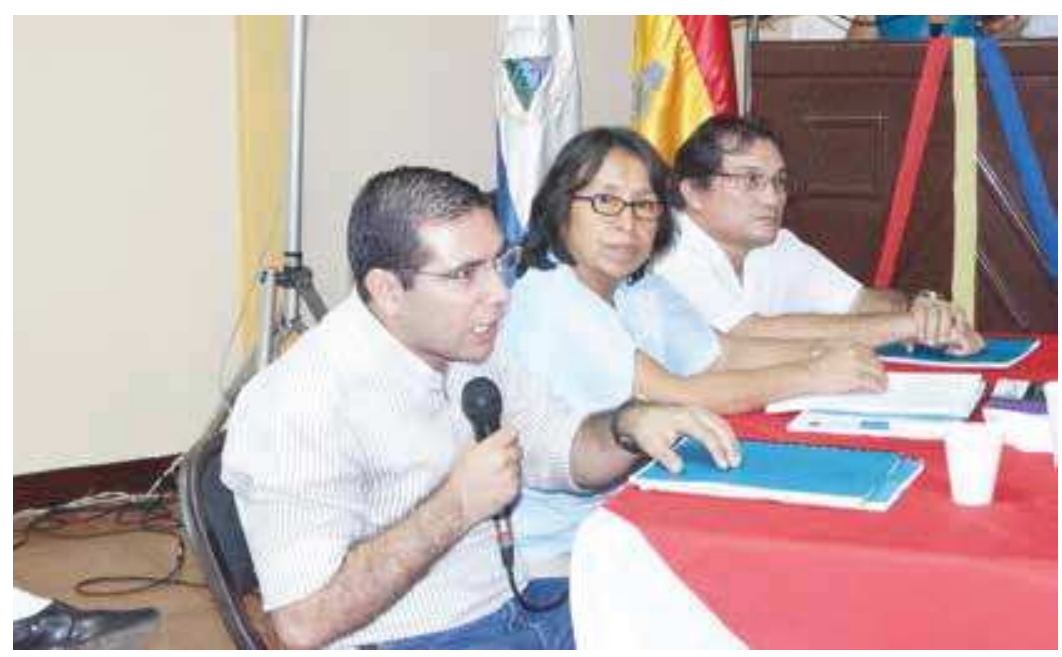

* MSc. Vicedecana Facultad de Humanidades y Ciencias Jurídicas 
bajo las modalidades establecidas en la Normativa General delaJUDC: Informes de Investigación científica, Ensayos, Proyectos de Desarrollo, Pre defensa de Monografías, Protocolos de investigación y Artículos Científicos, producción resultante de los trabajos de Cursos contemplados, en las Asignaturas que así lo estipulan, y en las áreas de Metodología de la investigación, investigación aplicada en correspondencia con las políticas y líneas de investigación de la Universidad, y con el Plan Nacional de Desarrollo Humano, definidas en la facultad y en los departamentos docentes.

El programa se desarrolló de forma intercalada entre los días 7 y 17 de octubre del corriente año en locales asignados para cada departamento docente. Una vez lograda la exposición y evaluación de los trabajos en cada unidad académica y finalizada el proceso de la evaluación correspondiente, se procedió a la selección de los primeros lugares, los cuales pasan a una segunda ronda, donde competirán por el primer lugar a nivel de la Facultad.

\section{Líneas de investigación reflejadas:}

Socioeconómica, ambiental, turística, jurídica, Derecho laboral, Derecho de Familia, Derecho Penal, histórica, arqueológica con énfasis en gestión para la conservación del Patrimonio Cultural, Geopolítica, Derechos territoriales y marítimos en el campo del Derecho Internacional, Pueblos indígenas, Culturas urbanas, lexicografía, estrategias comunicacionales, tradiciones, Asociatividad, Juventudes y participación Ciudadana, otros.

\begin{tabular}{|c|c|c|c|c|c|c|c|}
\hline \multicolumn{4}{|c|}{ TRABAJOS PRESENTADOS } & \multirow[b]{2}{*}{ Pre defensa } & \multirow[b]{2}{*}{ Protocolo } & \multirow[b]{2}{*}{ Proyectos } & \multirow[t]{2}{*}{ Obs. } \\
\hline Departamento & Inv. & ensayos & $\begin{array}{c}\text { Art- } \\
\text { Cient. }\end{array}$ & & & & \\
\hline Antropología & 24 & - & - & 8 & - & - & 32 \\
\hline Derecho & 3 & 3 & - & - & 4 & - & 10 \\
\hline Filosofía & - & 15 & - & & 8 & 2 & 25 \\
\hline Filología & 11 & $5-$ & - & 1 & 4 & 2 & 23 \\
\hline Geografía & 2 & - & - & 1 & 5 & 2 & 10 \\
\hline Historia & 11 & - & - & & & & 15 \\
\hline Psicología & 11 & - & & & & & 11 \\
\hline Trabajo Social & 4 & - & - & & & & 4 \\
\hline Total & & & & & & & 130 \\
\hline
\end{tabular}

De un total de 140 trabajos, se proyectaron 130 equivalente a un $93 \%$. 


\section{Estudiantes ganadores en la VIII JUDC Facultativa.}

\begin{tabular}{|c|c|c|c|c|c|c|}
\hline Lugar & Tema & Autores & Tutor & Inv. & Proy & Obs. \\
\hline 1ero & $\begin{array}{l}\text { Patrones } \\
\text { socioculturales } \\
\text { que inciden en la forma } \\
\text { de organización, relación } \\
\text { e intercambio de los } \\
\text { comerciantes del Mercado } \\
\text { Oriental. Managua } 2013\end{array}$ & $\begin{array}{l}\text { Norling Sabel } \\
\text { Solis Narvaez. }\end{array}$ & $\begin{array}{l}\text { Msc. Elvira } \\
\text { Maritza } \\
\text { Andino }\end{array}$ & $x$ & & Antropologia \\
\hline 2do. & $\begin{array}{l}\text { Tramitación y aplicabilidad } \\
\text { de los incidentes Promovidos } \\
\text { en la fase de ejecución de } \\
\text { sentencia penal }\end{array}$ & $\begin{array}{l}\text { Martha Karina } \\
\text { Jarquín } \\
\text { y José Alfredo } \\
\text { Herradora }\end{array}$ & $\begin{array}{l}\text { Msc. Ulises } \\
\text { Carballo }\end{array}$ & $x$ & & Derecho \\
\hline 3ro. & $\begin{array}{l}\text { Estrategia de Comunicación } \\
\text { con enfoque de riesgo; Barrio } \\
\text { Naciones Unidas. }\end{array}$ & $\begin{array}{l}\text { Jolany Karina } \\
\text { Delgado Ramos }\end{array}$ & $\begin{array}{l}\text { Esp. Lesbia } \\
\text { Bermúdez. }\end{array}$ & $X$ & & $\begin{array}{l}\text { Filología y } \\
\text { Comunicación }\end{array}$ \\
\hline 3ro. & $\begin{array}{l}\text { Avance de un diagnostico } \\
\text { participativo para la } \\
\text { elaboración de un Plan de } \\
\text { Mejora en el Municipio de } \\
\text { Santo Domingo. Chontales. }\end{array}$ & $\begin{array}{l}\text { Felipe E. } \\
\text { Mairena; } \\
\text { Alejandra R. } \\
\text { Pérez; Paola } \\
\text { Muñoz Ferrey y } \\
\text { Judith Obando } \\
\text { R. }\end{array}$ & $\begin{array}{l}\text { Dra. Jilma } \\
\text { Romero. }\end{array}$ & $x$ & & Historia \\
\hline
\end{tabular}

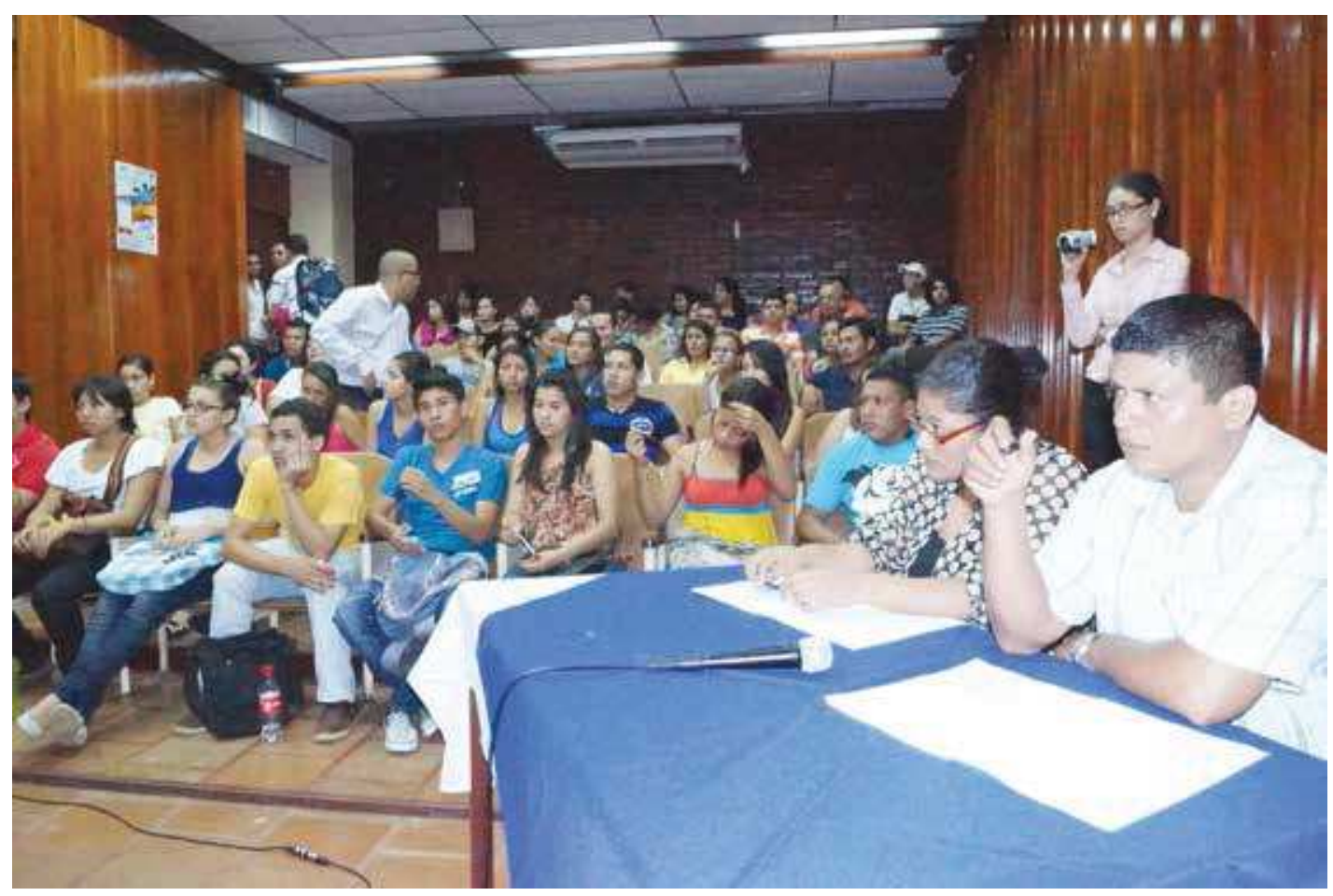




\section{Fortalezas:}

$\checkmark$ La inclusión de la JUDC en la Planificación Operativa Anual de la Facultad.

$\checkmark$ La estrategia de organizar un Taller para jurados y docentes involucrados en la JUDC, el día 5 de octubre, se discutió la normativa de la JUDC y se analizaron los Instrumentos de evaluación. Fue un espacio importante para unificar criterios sobre la metodología y guías de evaluación para trabajos de investigación, proyectos $\mathrm{y}$ ensayos.

$\checkmark$ En la inauguración de la VIII Jornada Facultativa, se contó con la presencia del equipo del decanato, por ser un colectivo que de manera sistemática, se reúne para tratar todos los ejes relacionados con el quehacer de la facultad en el campo de la docencia, investigación y extensión social.

$\checkmark$ La organización de la JUDC Facultativa, conllevo un esfuerzo y compromiso institucional, aglutinando al sector docente, administrativo y estudiantil lo que garantizo con eficiencia la presentación de los trabajos (horarios y distribución de los miembros de los jurados por cada área)

\section{Sugerencias:}

< Articular temas de interés a investigar en correspondencia con las líneas definidas y que sean orientados por los docentes en sus respectivas unidades académicas.

\& Dar seguimiento por parte de la Dirección de Departamento y los Coordinadores de investigación a los trabajos de curso, para estimular más la motivación y participación de los estudiantes.

$<$ Promover estrategias que posibiliten una premiación atractiva para establecer bases articuladas de interés por la investigación que a su vez se plasme en una mejor calidad en la participación y presentación de los estudiantes.

\& Planificar mayor presupuesto para esta jornada 


\section{Anexos}

\section{Departamento de Antropología}
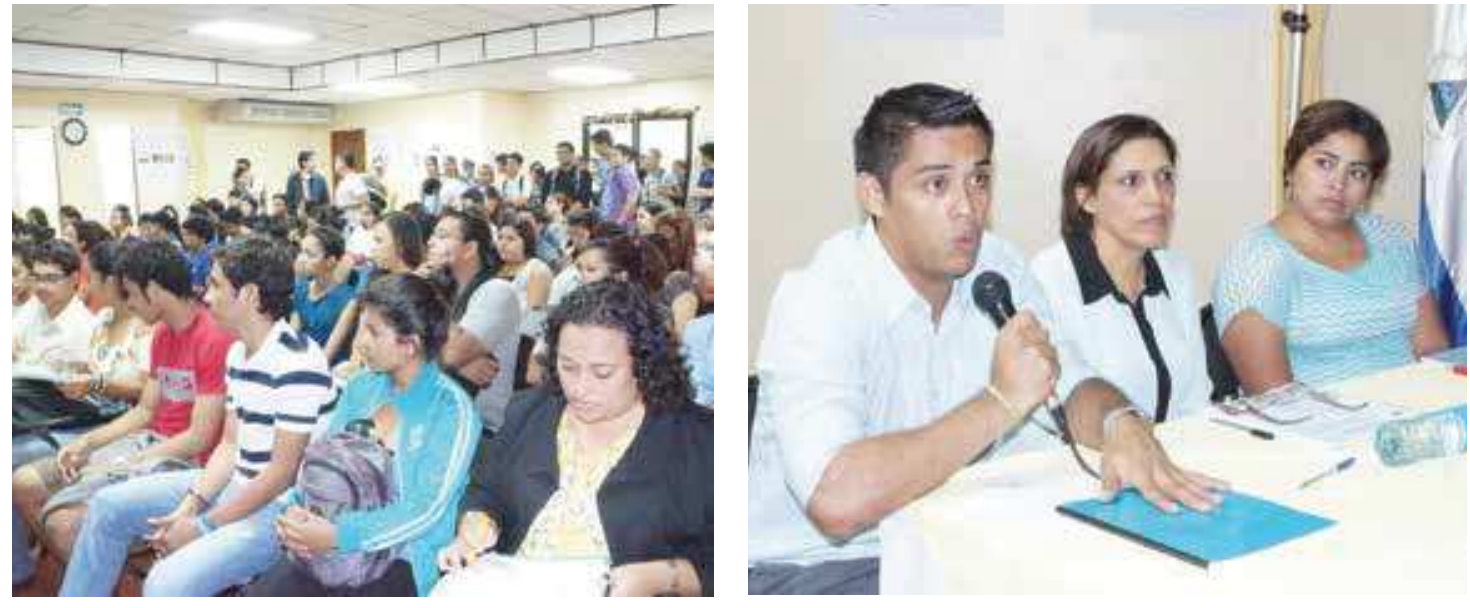

\section{Departamento de Historia:}
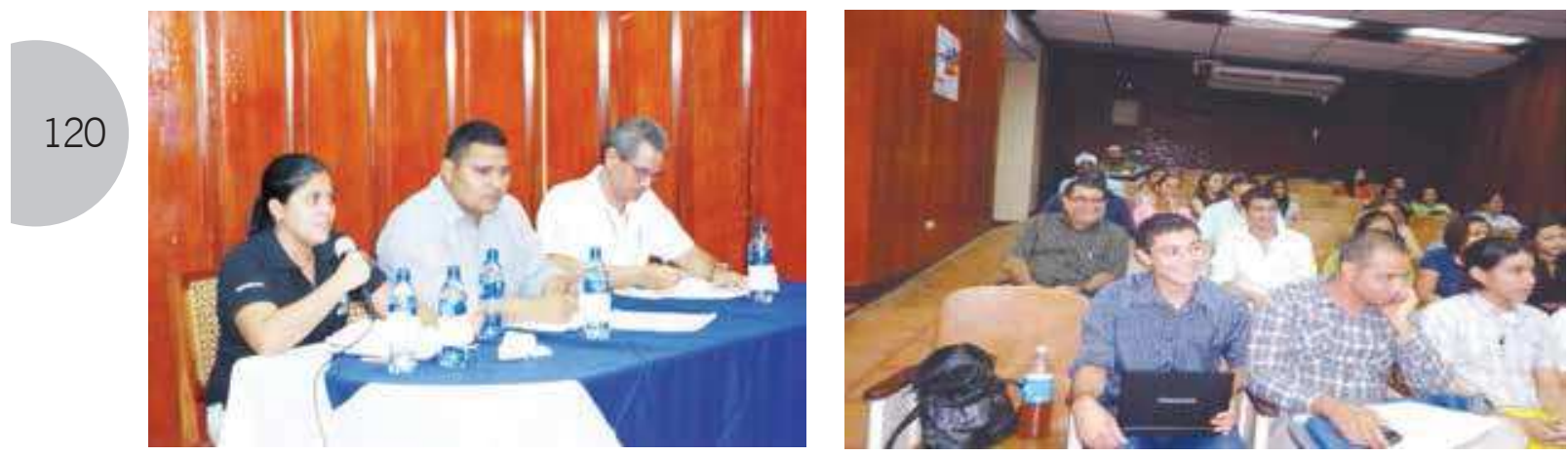

Departamento de Derecho :
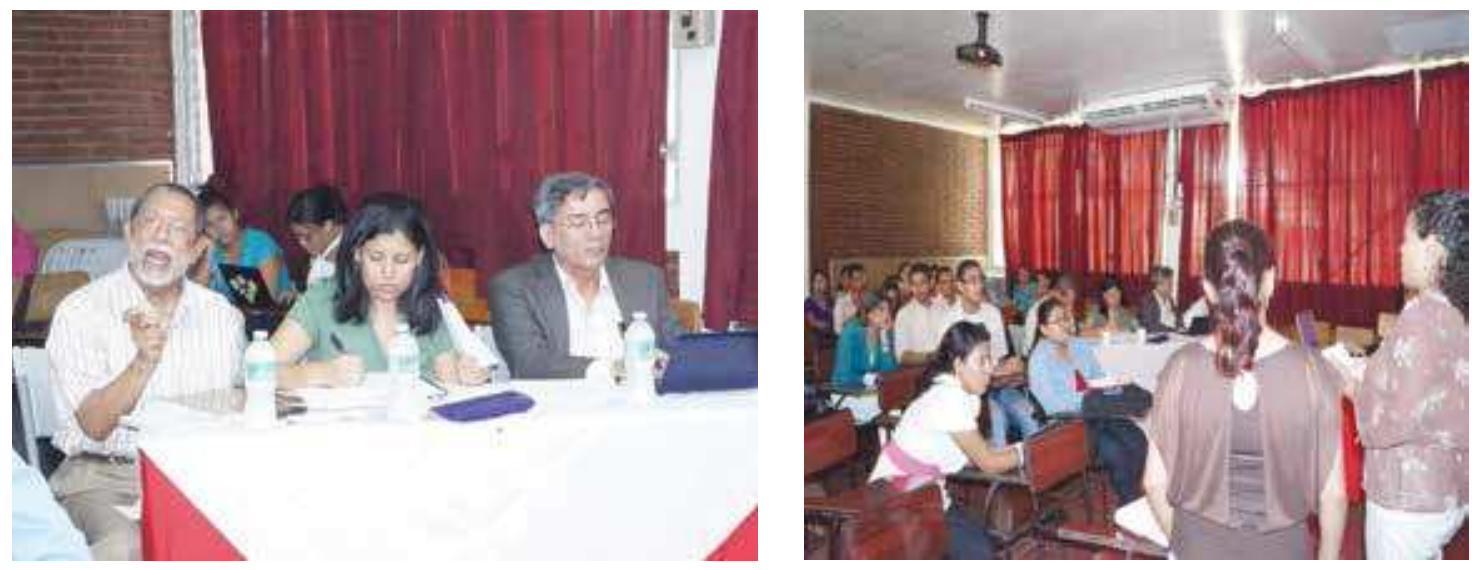


\section{Departamento de Filologia :}

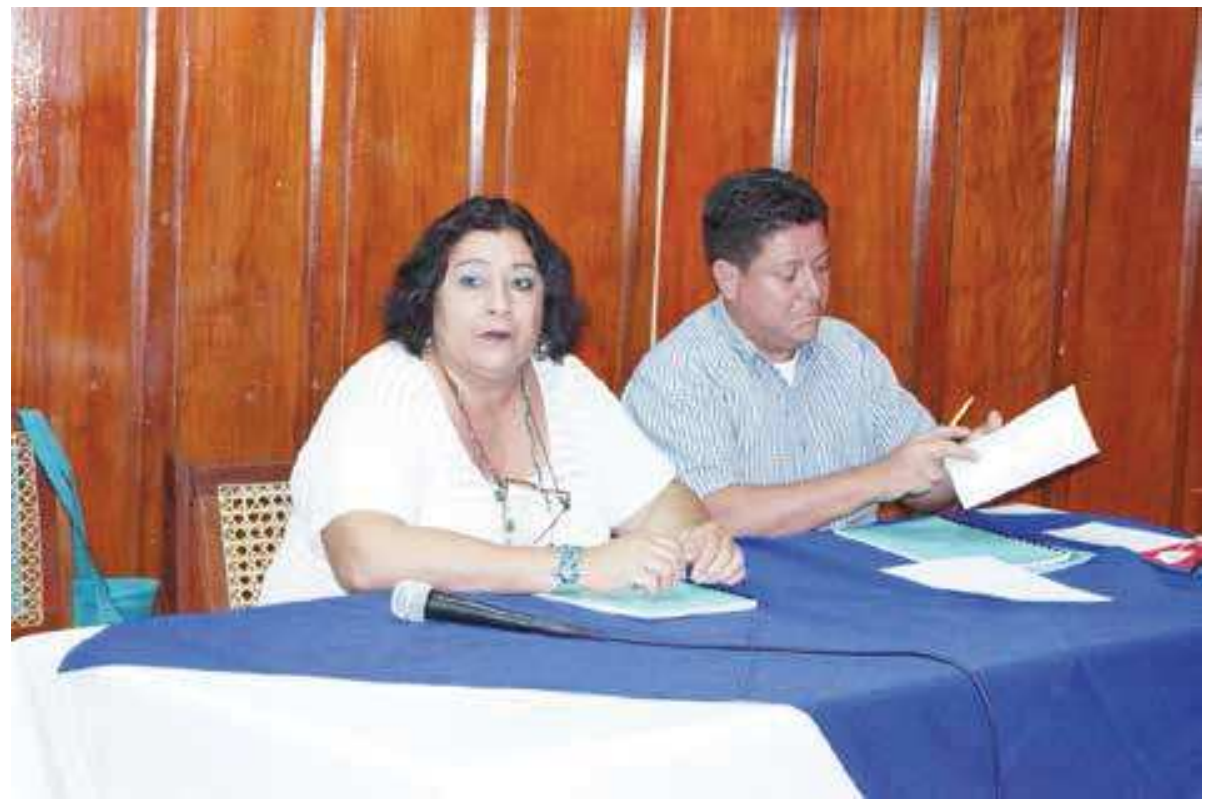

Departamento de Filosofia :
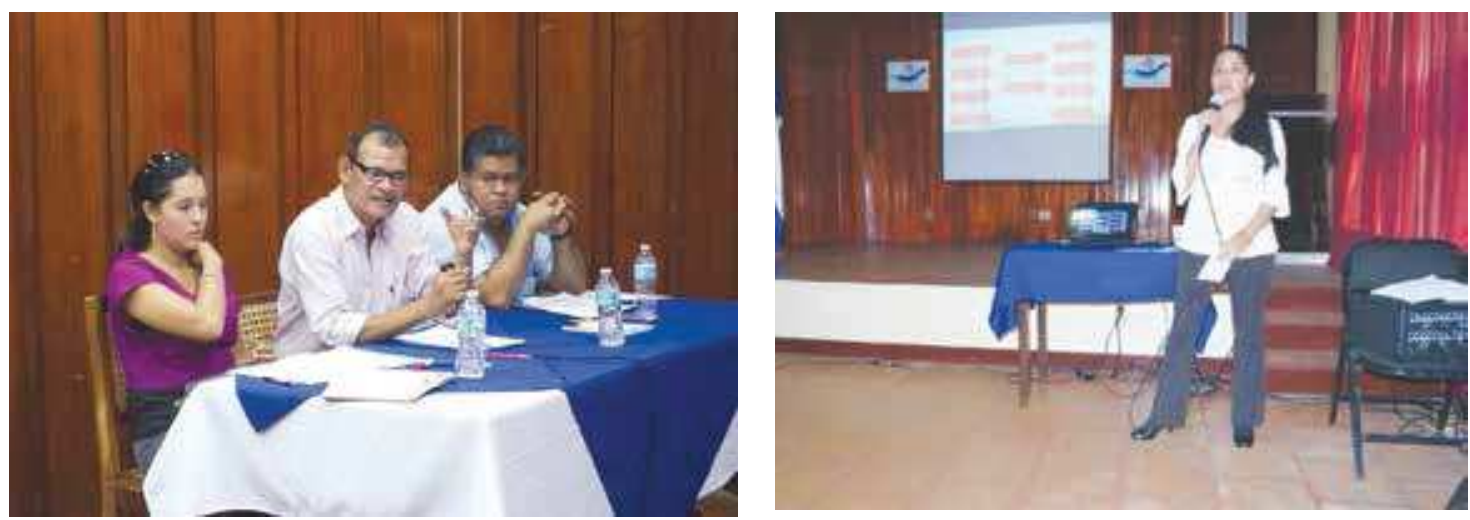

\section{Departamento de Geografia :}
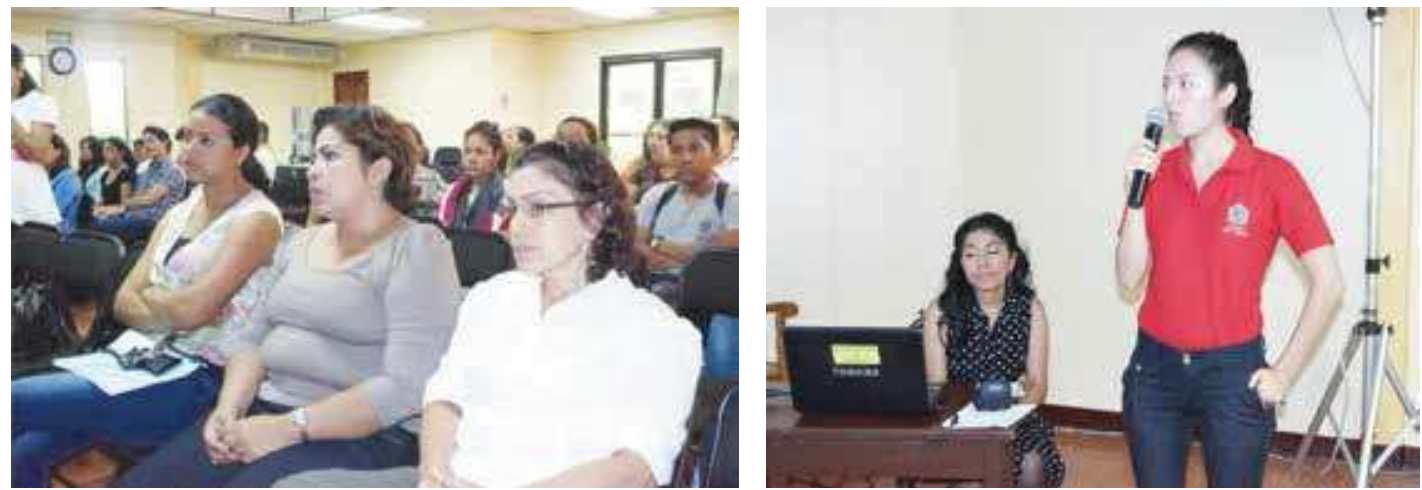

Gloria Argentina López Alvarado

VIII JORNADA UNIVERSITARIA DE DESARROLLO CIENTÍFICO (JUDC) 2013 


\section{Departamento de Psicologia:}
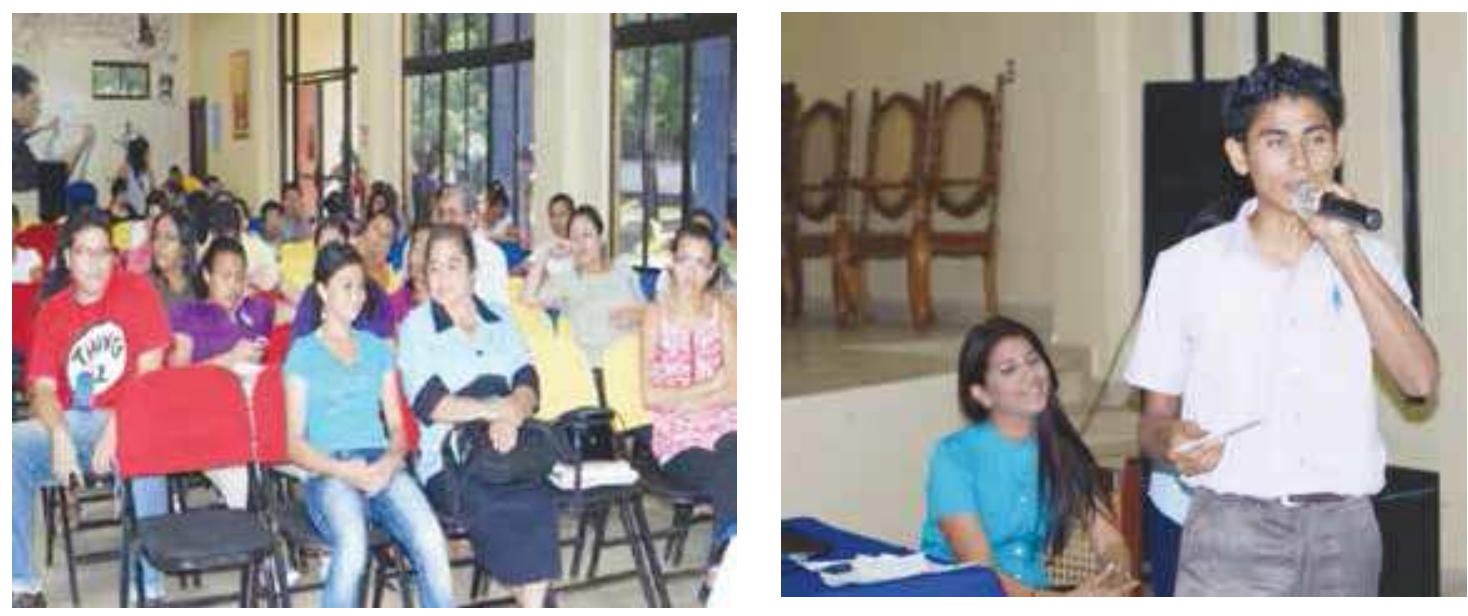

\section{Carrera de Trabajo Social :}

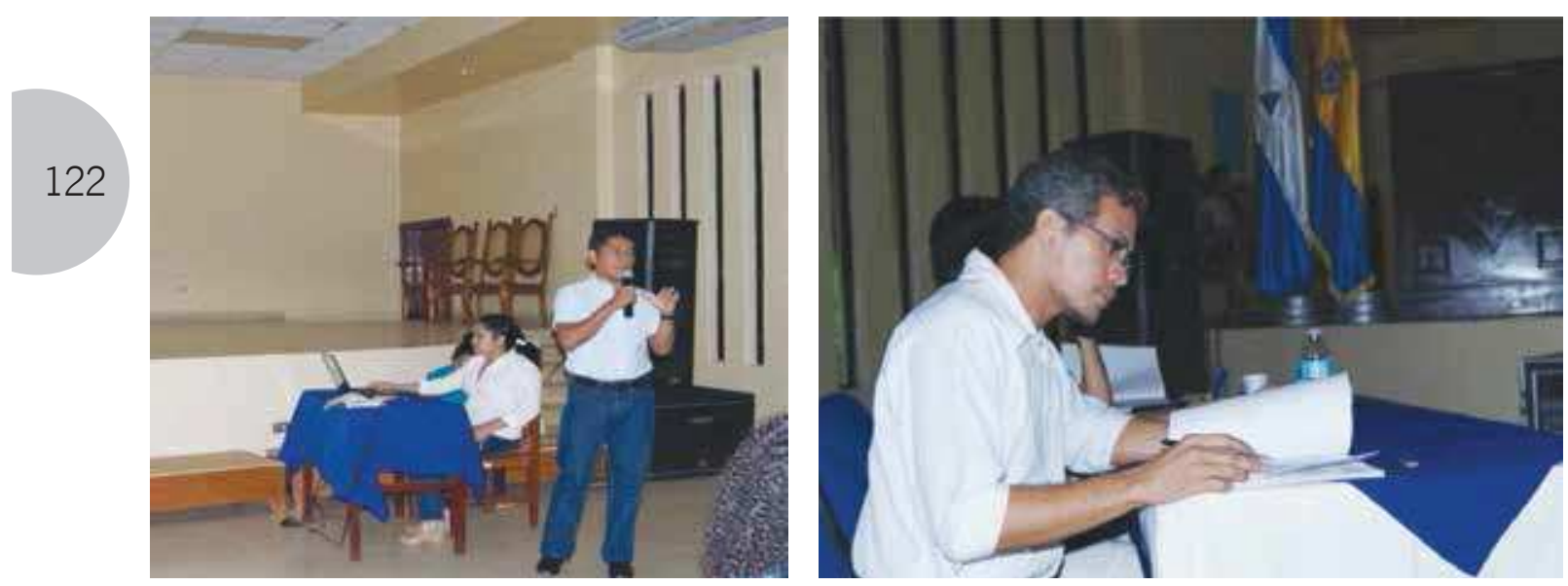

